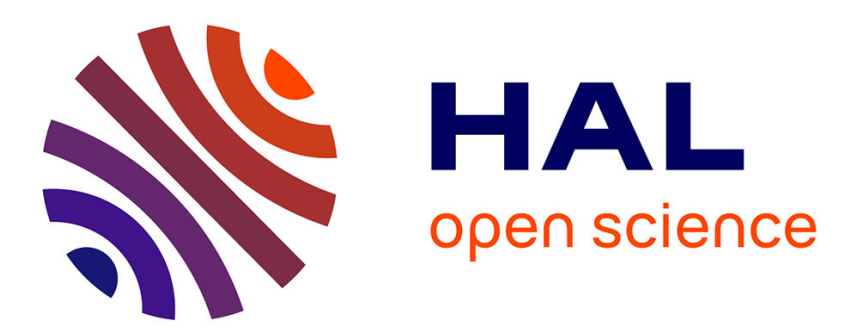

\title{
Co-construction d'un design logistique des circuits courts en territoire rural : le cas de Saint-Flour Communauté
}

Virginie Noireaux, François Cassière

\section{To cite this version:}

Virginie Noireaux, François Cassière. Co-construction d'un design logistique des circuits courts en territoire rural : le cas de Saint-Flour Communauté. Question(s) de Management, 2021, $\mathrm{n}^{\circ} 33$ (3), pp.75-87. 10.3917/qdm.213.0075 . hal-03420491

\section{HAL Id: hal-03420491 \\ https://hal.science/hal-03420491}

Submitted on 9 Nov 2021

HAL is a multi-disciplinary open access archive for the deposit and dissemination of scientific research documents, whether they are published or not. The documents may come from teaching and research institutions in France or abroad, or from public or private research centers.
L'archive ouverte pluridisciplinaire HAL, est destinée au dépôt et à la diffusion de documents scientifiques de niveau recherche, publiés ou non, émanant des établissements d'enseignement et de recherche français ou étrangers, des laboratoires publics ou privés. 
Co-construction d'un design logistique des circuits courts en territoire rural : le cas de Saint-Flour Communauté

NOIREAUX Virginie, Maître de conférences HDR, CleRMa (EA 3849), virginie.noireaux@uca.fr

CASSIERE François, Maître de conférences, CleRMa (EA 3849), francois.cassiere@ uca.fr 


\title{
Co-construction d'un design logistique des circuits courts en territoire rural : le cas de Saint-Flour Communauté
}

\section{Résumé}

Le développement économique dans les zones rurales est au cœur de bien des politiques publiques. Comment stimuler le tissu local et remettre du dynamisme dans des zones rurales à faibles populations et vigueur commerciale limitée? Une zone agricole de moyenne montagne auvergnate tente de relever ce challenge en proposant de bâtir une démarche collective parmi des producteurs agricoles. Le choix des circuits courts permettant d'accroitre ses marges est une option intéressante pour qui sait s'organiser et être efficace dans ce mode de distribution. Les questionnements des producteurs mettent en évidence l'importance d'un design logistique sur mesure servant les intérêts de toutes les parties prenantes. Mobilisant une démarche d'étude qualitative en recherche-intervention, les chercheurs mettent en relief une organisation logistique cohérente des circuits courts dans le territoire. Pour obtenir cette organisation, il faut prendre en compte les spécificités géographiques du territoire, les contraintes de chacune des filières, les organisations logistiques déjà présentes et les attentes des acteurs du territoire. Les différents va et vient avec les acteurs ont conduit à la construction d'un modèle organisationnel porté par la collectivité, optimisant la logistique, et par deux solutions techniques permettant de répondre aux enjeux des acteurs. Au-delà des aspects techniques, ce projet a permis aux acteurs du territoire de se projeter dans davantage de coopération, en préservant les intérêts individuels de chacun.

Mots clé : canaux courts, agriculture de moyenne montagne, construction collective, solutions logistiques.

\begin{abstract}
Economic development in rural areas is at the heart of many public policies. How can we stimulate the local infrastructure and restore dynamism in rural areas with low populations and limited commercial vitality? An agricultural area in the mid-mountain region of Auvergne is trying to face this challenge by proposing to build a collective approach among farming producers. The choice of short circuits to increase margins is an interesting option for those
\end{abstract}


who know how to organize themselves and be efficient in this distribution mode. The producers' questions highlight the importance of a tailor-made logistic design serving the interests of all stakeholders. Using a qualitative research-intervention study approach, the researchers highlight a coherent logistical organization of short circuits in the territory. To obtain this organization, it is necessary to take into account the geographical specificities of the territory, the constraints of each of the activities, the logistic organizations already present and the expectations of the territorial stakeholders. The various exchanges with the actors led to the construction of an organizational model supported by the community, optimizing logistics, and by two technical solutions allowing to answer the actors' challenges. Beyond the technical aspects, this project allowed the actors of the territory to project themselves in a more cooperative way, while preserving the individual interests of each one.

Key words: short channels, mid-mountain agriculture, collective construction, logistics solutions. 


\section{Introduction}

Les circuits courts ne sont pas nouveaux en France, mais ont souffert, jusqu'à récemment, d'un manque de reconnaissance (Chiffoleau et Paturel, 2016). Leur recrudescence est liée à de nombreux facteurs comme les peurs alimentaires, les nouvelles attentes des consommateurs en matière de qualité, le développement des valeurs associés aux projets «alternatifs»... (Maréchal, 2008). Cet intérêt se traduit dans les chiffres. Une étude de l'Agreste (2016) indiquait que $30 \%$ des exploitations agricoles commercialisait une partie de leur production en vente directe en 2016 dont $11.5 \%$ avec un seul intermédiaire. Si le développement des circuits courts est largement plébiscité par les producteurs et les consommateurs, leur organisation ne se réalisent pas forcément de façon aisée. Le pas n'est pas facile à franchir de «l'intention » au savoir « comment le faire ? ». En effet, le développement des circuits courts demande une réorganisation totale de la distribution, ce qui impacte largement les aspects marketings et logistiques (Noireaux et Ralet, 2019).

La question logistique est souvent évoquée dans la littérature, mais son design reste un enjeu stratégique majeur du développement des circuits courts. Le design logistique représente à la fois l'organisation et les solutions techniques retenues. Il doit être adapté aux caractéristiques des acteurs et du territoire étudié. Pour chercher le meilleur design logistique, les acteurs d'un territoire doivent remplir trois conditions (Pacciarotti et Torregiani, 2021). Ils doivent être ouverts à des innovations organisationnelles et techniques. Ils doivent accepter des collaborations entre différentes parties prenantes, et ils doivent développer des coopérations avec des chercheurs qui sont en mesure d'analyser la situation et de proposer des solutions. C'est dans ce cadre que nous avons effectué une recherche-action sur le territoire de Saint-Flour Communauté. Ce travail a pour ambition de comprendre les pratiques et les problématiques liées au développement des circuits courts sur le territoire, et de co-construire une solution logistique pertinente. Ainsi, nous cherchons à comprendre quel design logistique peut permettre aux acteurs d'un territoire rural de développer les circuits courts.

Pour ce faire, nous présenterons une revue de littérature, puis nous exposerons la méthodologie et les spécificités du territoire étudié. Enfin, nous présenterons les résultats de l'étude.

\section{Les enjeux de la logistique des circuits courts dans les territoires ruraux}


Le développement des circuits courts alimentaires est porteur d'enjeux multiples auprès des différentes parties prenantes des territoires, notamment dans les territoires ruraux où l’organisation logistique doit répondre aux spécificités liées à la ruralité.

\subsection{Les enjeux des circuits courts pour les acteurs des territoires}

Les circuits courts alimentaires représentent la vente de la production d'un agriculteur directement au client ou avec un seul intermédiaire. Si la question de la distance entre producteurs et consommateurs est également évoquée par certains auteurs (Blanquart et al., 2009), elle ne fait pas consensus au sein de la communauté scientifique (Pacciarotti et Torregiani, 2021). C'est pourquoi, nous retiendrons uniquement dans cet article la notion restreinte d'intermédiaires (1 maximum) sans restriction spatiale.

Les circuits courts sont des organisations qui abritent une diversité d'organisations (schéma 1), d'acteurs et d'ambitions. Ils renvoient à une réalité multiple, qui se traduit par une vingtaine de modalités de vente (Chiffoleau et Paturel, 2016) engendrant chacune enjeux et contraintes spécifiques (voir les travaux de Maréchal, 2008). A titre d'exemple, la procédure d'installation sur un marché peut être rapide, mais la cohabitation de multiples acteurs et/ou les intempéries peuvent être des entraves à l'activité du producteur. Pour les ventes en restauration collectives, les débouchés économiques sont importants, mais ce canal demande une bonne connaissance des parties prenantes et des réglementations liées au marché public.

Schéma 1 : Typologie des circuits courts 


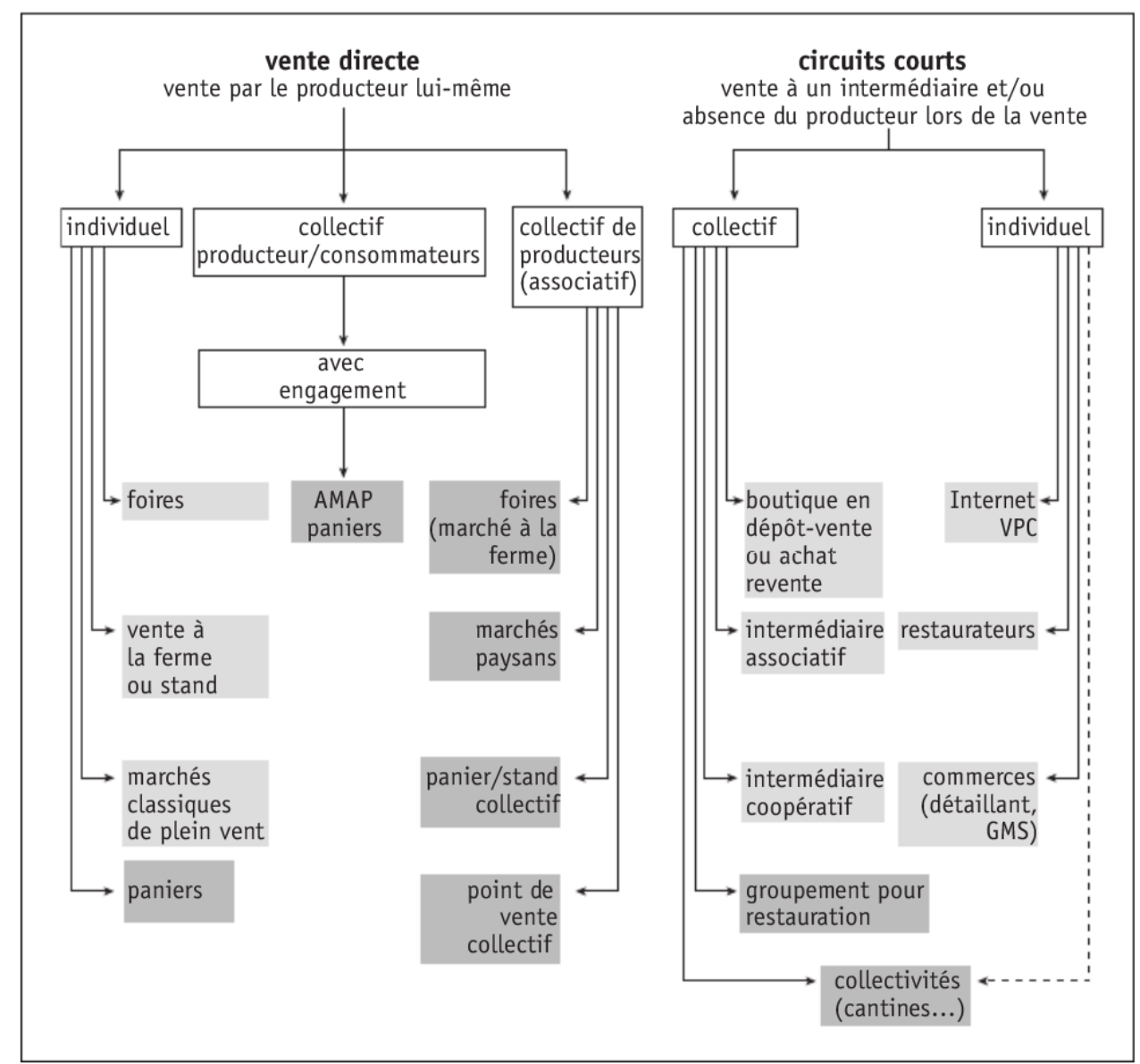

Source : Maréchal (2008)

Pour le producteur le circuit court est l'opportunité de diversifier sa distribution, jusque-là majoritairement tournée vers les grossistes. Cela lui permet de répondre à deux enjeux. Le premier est financier en offrant une meilleure rémunération (Chiffoleau, 2019). Le second est lié à une meilleure intégration sociale. Du fait d'un renforcement des liens agriculture alimentation, et production - consommation, les échanges entre producteurs, clients et diverses parties prenantes sont multipliés (Chiffoleau, 2019). Les circuits courts conduiraient alors à un certain bien-être subjectif, traduit par l'accroissement de plusieurs facteurs enchevêtrés : l'augmentation des revenus financiers, les échanges d'informations et d'expériences avec diverses parties prenantes, et les relations de confiance entre producteurs et consommateurs (Bonomelli et Roudart, 2019). Ils seraient également le vecteur permettant une meilleure viabilité et transmission des exploitations (Noireaux et Ralet, 2019). Pour le client final le recours à des circuits courts répond à un souhait de garantie sur la qualité des aliments qu'il consomme, et une recherche d'identité (Chiffoleau, 2019). Les produits alimentaires deviennent alors un vecteur privilégié de distinction et d'affirmation d'une 
identité culturelle (Munzele Munzimi, 2013).

Le développement des circuits courts a des conséquences sur l'ensemble des acteurs (Moati 2018). De l'amont à l'aval, chacun est interrogé sur ses pratiques et ses opportunités de développement. Noireaux et Ralet (2019) ont montré qu'à l'échelle d'un territoire rural, le développement des circuits courts fait largement consensus entre les différentes parties prenantes. Pour les producteurs agricoles, ancrés au sein de territoires très retirés, les enjeux sont liés à la maitrise des coûts et à la logistique. En effet, le passage en circuits courts peut engendrer des difficultés chez eux en matière de ressources et de compétences pour optimiser leur logistique (Paciarotti et Torregiani, 2021). Au niveau d'un éventuel intermédiaire (commerçant, artisan...), il doit apporter des réponses logistiques et commerciales à ses clients (qualité des produits, faible taux de ruptures...) pour permettre la viabilité et/ou le développement de son activité. Les consommateurs souhaitent avoir davantage d'informations sur la provenance des produits (région, village...), et accéder à une grande diversité alimentaire en limitant leurs transports (Vaillant et al., 2017). Enfin, pour les acteurs institutionnels, le développement des circuits courts est un enjeu politique (développement ou / et au maintien de l'attractivité du territoire pour les acteurs économiques), de maîtrise du coût de certains services publics (cantine...), et/ou de choix d'investissements (routes...) dans un contexte où les recettes locales de l'activité économique et les dotations étatiques sont de plus en plus limitées. Le tableau 1 suivant présente les différents enjeux des circuits courts pour chaque type d'acteur.

Tableau 1 : Enjeux des circuits courts pour les acteurs d'un territoire 


\begin{tabular}{|c|c|c|}
\hline Acteur & $\begin{array}{c}\text { Enjeux du développement des circuits } \\
\text { courts }\end{array}$ & Auteurs \\
\hline \multirow[t]{4}{*}{ Producteur } & Meilleure rentabilité & $\begin{array}{l}\text { Chiffoleau, } 2019 \text {; Bonomelli et } \\
\text { Roudart, } 2019 \text {; Requier-Desjardins et } \\
\text { Roudart. } 2019 \text {; Mundler et Laughera. } \\
2015\end{array}$ \\
\hline & $\begin{array}{l}\text { Transmission/ maintien des } \\
\text { exploitations }\end{array}$ & $\begin{array}{l}\text { Maréchal, } 2008 \text {; Noireaux et Ralet, } \\
2019 \text {; Mundler et Laughera, } 2015\end{array}$ \\
\hline & Coopération entre producteurs & Maréchal, 2008 ; \\
\hline & $\begin{array}{l}\text { Reconnaissance et valorisation du métier } \\
\text { de paysan }\end{array}$ & $\begin{array}{l}\text { Maréchal, } 2008 \text {; Chiffoleau, 2017, } \\
2019 \text {; Bonomelli et Roudart, 2019; } \\
\text { Requier-Desjardins et Roudart. } 2019 \text {; } \\
\text { Mundler et Laughera, 2015 }\end{array}$ \\
\hline \multirow[t]{2}{*}{ Consommateur } & $\begin{array}{l}\text { Information sur la production (lieu, } \\
\text { techniques...) }\end{array}$ & $\begin{array}{l}\text { Maréchal, } 2008 \text {; Chiffoleau, } 2019 \text {, } \\
\text { Vaillant et al., } 2017 \text {; Noireaux et } \\
\text { Ralet. } 2019\end{array}$ \\
\hline & Explication et justification du coût & $\begin{array}{l}\text { Maréchal, } 2008 \text {; Chiffoleau, } 2019, \\
\text { Bonomelli et Roudart, } 2019\end{array}$ \\
\hline Institution & $\begin{array}{l}\text { Développement local : accès aux } \\
\text { commerces et services (dont restauration } \\
\text { collective), développement des } \\
\text { investissements... }\end{array}$ & $\begin{array}{l}\text { Maréchal, } 2008 \text {; Noireaux et Ralet, } \\
2019 \text {; Mundler et Laughera, } 2015\end{array}$ \\
\hline $\begin{array}{l}\text { Acteur } \\
\text { intermédiaire } \\
\text { (distributeur, } \\
\text { association ou } \\
\text { autre...) }\end{array}$ & $\begin{array}{l}\text { Apporter des réponses commerciales et } \\
\text { logistiques aux clients } \\
\text { Viabilité/ développement de } 1^{\text {'activité }}\end{array}$ & $\begin{array}{l}\text { Maréchal, } 2008 \text {; Noireaux et Ralet, } \\
2019\end{array}$ \\
\hline
\end{tabular}

Elaboration personnelle

Autour ces enjeux, les problématiques logistiques apparaissent comme un point névralgique. En effet, la logistique des circuits courts entraine des coûts cachés (Mundler et Laughera, 2015 ; Vaillant et al. 2017, Noireaux et Ralet, 2019) : contre-performance des émissions de gaz à effets de serre liés aux transports (Vaillant et al. 2017) et multiplication des tâches associées (organisation des expéditions et/ou transport des produits, emballage dans des lots de taille parfois réduite...). Face à ce challenge, les acteurs doivent alors trouver des solutions innovantes pour optimiser leurs organisations logistiques (Vaillant et al. 2017 ; Mundler et Laughera, 2015 ; Noireaux et Ralet, 2019).

\subsection{Problématiques logistiques des circuits courts en territoire rural}

Les réseaux territoriaux d'organisation ont été développés par Alfred Marshall dès 1919. Depuis, ils se sont enrichis d'adaptations, notamment en géographie économique, en insistant sur le fait que la proximité géographique est génératrice d'échanges informels, et peut fonder des mécanismes de coordination horizontale (Aoki, 1990). Cette dimension territoriale permet donc de relier les systèmes logistiques aux modèles productifs (Masson et Petiot, 2013). Le territoire peut être définit comme «un système complexe évolutif qui associe un 
ensemble d'acteurs d'une part, l'espace géographique que ces acteurs utilisent, aménagent et gèrent d'autre part»(Moine, 2006, p.126). En logistique, la notion de territoire est généralement appréhendée comme un support qui offre des ressources spécifiques (routes...) et qui constitue des contraintes (accessibilité, climat, réglementations ...) sur lequel les acteurs doivent optimiser les flux. Cette analyse intègre les deux logiques qui fondent la notion de territoire (Raulet-Clauset, 2014) : le territoire prescrit, qui renvoie à des définition d'action et de politique publique (interdiction de circulation, aménagement des routes...), et le territoire construit qui émerge d'usages et d'appropriations par des acteurs (organisation des flux).

Si les limites des circuits courts sont nombreux : focalisation sur des marchés de niche ; production faible (variété et quantité); difficultés d'organisation et de coordination ; coûts logistiques élevés ; accès restreint aux économies d'échelle; ressources et compétences limités en communication et marketing; «burnout» possible des producteurs qui doivent endosser de multiples rôles..., le design et les innovations logistiques sont susceptibles de d'améliorer considérablement les impacts négatifs (Paciarotti et Torregiani, 2021). Les solutions logistiques permettent de limiter considérablement les impacts écologiques (réduction des émissions de $\mathrm{CO} 2$ par une meilleure organisation permettant des transports complets, utilisation d'outils moins polluant...), sociaux (allègement de tâches chez l'agriculteur comme l'emballage ou le transport, développement de compétences pour organiser les flux...) et économique (optimisation du coût du transport, mutualisation permettant de vendre davantage de variété et de quantité).

Au-delà des limites des circuits courts évoquées dans la littérature, la ruralité du territoire peut également être source de difficultés. Ainsi, un éloignement des principaux axes routiers peut fortement augmenter les coûts de transport et les délais de disposition. A l'échelle d'un territoire rural, les clients sont disséminés çà et là, ce qui complexifie la logistique des producteurs (Noireaux et Ralet, 2019). Ils se retrouvent face à des difficultés liées aux « premiers kilomètres » qui représentent le coût du premier maillon de la chaîne logistique partant du producteur et à destination d'un autre acteur du canal. Ces difficultés peuvent se traduire par des temps de transport plus importants sur le réseau routier secondaire, des déplacements devenant aléatoires en moyenne montagne (enneigement, gel... congestions des routes touristiques à certaines périodes), des prix de transport élevés du fait de l'éloignement des grands axes ou encore des difficultés de trouver un transporteur qui veuille se déplacer.

Force est de constater que la coordination des flux (rentabilité, normalisation...) est plus 
complexe dans un contexte rural. Il faut donc une très bonne connaissance du territoire pour pouvoir adapter au mieux son offre (Pouzenc, 2012). Ceci met le pilotage logistique, au cœur de la réflexion, notamment celle sur les mécanismes de mutualisation entre producteurs. C'est un enjeu-clé du développement des circuits courts dans les territoires ruraux.

Ce pilotage reste une réelle problématique. S'il a été fréquemment mentionné que les agriculteurs n'ont pas les compétences nécessaires (Cassière et Noireaux, 2020 ; Noireaux et Ralet, 2019), la prise en charge de cette variable via un intermédiaire privé est rarement bien vécue par les producteurs (Paciarotti et Torregiani, 2021). Une solution plus fructueuse et adéquate peut émaner d'un échange collectif entre les différentes parties prenantes (Cassière et Noireaux, 2020). Cette réflexion commune permet de dépasser certaines limites individuelles (manque de ressources, de compétences, notamment logistiques) ou la contrainte imposée par un acteur trop puissant. Même si cette solution semble séduisante sur le papier, sa mise en place et son développement connaissent de nombreuses entraves : difficultés de gestion (lenteurs des décisions, scepticisme/opportunisme des partenaires, défaut de communication, ...), conflits interpersonnels, absence de certaines parties prenantes clés dans le projet, absence de vision commune... (Dari et Paché, 2015).

Le pilotage des activités communes semble être un point névralgique pour la mise en œuvre du design logistique. Le défi est lié à la mise en place et à l'application de règles communes, ce qui doit être réalisé par un acteur pivot neutre, qui permet d'organiser la logistique en préservant la concurrence entre les organisations. Les organisations qui coopèrent n'ont alors pas accès à certaines données de leurs concurrents (noms des clients, quantités expédiées...). Dans la littérature en logistique, cet acteur pivot est généralement le Prestataire de Services Logistique (PSL) (Rouquet et Vauché, 2015). Mais d'autres acteurs peuvent également prendre ce rôle, par exemple, des associations de producteurs (Noireaux et Poirel, 2014). Si les stratégies collectives sont délicates à mettre en œuvre, elles peuvent se révéler très efficaces.

\section{Méthodologie : recherche-intervention au sein d'un territoire rural}

Nous avons utilisé un protocole de recherche-intervention, qui permet de concevoir des connaissances simultanément pour et par l'organisation et les chercheurs, dans une logique transformative (Krief et Zardet, 2013). Celle-ci s'est déroulée sur le territoire de Saint-Flour communauté. 


\subsection{Le protocole méthodologique de recherche-intervention}

L'étude du cas Saint-Flour Communauté, s'inscrit dans le cadre global du projet LEADER dont l'objet est de construire collectivement des solutions logistiques avec les agriculteurs. Ce travail est très fortement soutenu par la Communauté de communes qui souhaite s'investir à long terme dans une organisation efficace des flux pour de donner de la visibilité aux acteurs du territoire. L'objectif est donc de construire collectivement un système logistique adapté aux pratiques des acteurs et aux caractéristiques du territoire, qui permet de répondre aux spécificités des différentes filières présentes (fromage, viande, légumes et miel).

Entre janvier 2019 et septembre 2020, 8 réunions collectives (producteurs, collectivités et chercheurs) et 30 entretiens semi-directifs ont été conduits auprès d'acteurs du monde agricole sur le territoire (Tableau 2). Parallèlement, une observation non participante a été faite lors des entretiens à travers la visite des sites agricoles. Ceci nous a permis d'apprécier les distances, les dispositifs liés à l'organisation de la logistique individuelle (superficie, aménagement, matériel de stockage, véhicule de livraison...). Les répondants sont issus de quatre filières représentant l'ensemble des activités agricoles de ce territoire. Les répondants sont majoritairement des exploitations de petite et moyenne taille.

Les réunions collectives se sont déroulées pendant toute la durée de l'étude et ont duré chacune entre $2 \mathrm{~h}$ et $2 \mathrm{H} 45$. Elles se déroulaient en deux temps. Dans un premier temps, les chercheurs restituaient les données collectées lors des entretiens et des visites. Puis, ils proposaient différents designs logistiques (issues des entretiens et des connaissances des chercheurs) au collectif (17 agriculteurs, la Communauté de communes et la Chambre d'agriculture). Pour constituer le collectif, la collectivité a contacté personnellement l'ensemble des agriculteurs sur le territoire afin de présenter le projet. Sur les 68 exploitants contactés, 17 se sont engagés contractuellement à suivre le projet, 34 ont acceptés de réaliser des entretiens (4 n'ont pas pu être conduits). De plus, l'ensemble des agriculteurs étaient invités à toutes les réunions collectives. Certains s'agrégeaient ponctuellement au «noyau contractuel » en fonction de leurs disponibilités. Les architectures logistiques proposés ont été largement débattues par les acteurs tant sur les plans organisationnels que techniques. Ces allers-retours réguliers préconisés par Moisdon (2015) ont permis d'impliquer les acteurs dans la réflexion, de pouvoir gérer le décalage des représentations, et de faire émerger un modèle final largement consensuel. Un guide d'entretien a été construit pour la conduite des 
interviews. Les entretiens d'une durée moyenne de 50 minutes environ représentent $27 \mathrm{~h} 30$ d'enregistrement et 360 pages de verbatim codifiés et catégorisés à l'aide logiciel QSR Nvivo 12 (527 pages de codage). Le codage des entretiens a suivi les thèmes et les sous-thèmes du guide d'entretien : description de l'activité, communication et information vers les clients, caractéristiques des flux, stockage et livraison, et enfin perception pour le futur. Un double codage a été réalisé. Ce guide avait préalablement été débattu avec les acteurs lors de la première réunion collective. Ces items permettant de comprendre les organisations logistiques actuelles, d'accompagner les acteurs dans une réflexion sur le futur envisagé, et de faire émerger des idées.

Tableau 2 : Structure de l'échantillon

\begin{tabular}{|c|c|c|c|}
\hline Nature de l'élevage & $\begin{array}{c}\text { Capacité de } \\
\text { production (an) }\end{array}$ & $\begin{array}{l}\text { Nombre de } \\
\text { personne sur } \\
\text { l'exploitation }\end{array}$ & $\begin{array}{l}\text { Remarques relatives à } \\
\text { l'ancienneté }\end{array}$ \\
\hline Chèvres/ brebis & $14000-15000$ litres & 2 & Reprise par la fille récente \\
\hline Vaches & $35000-40000$ litres & 3 & \\
\hline Coopérative (vache) & 9 millions de litres & 50 agriculteurs & \\
\hline Coopérative (vache) & 7 millions de litres & 40 agriculteurs & \\
\hline Vaches & 650000 litres & 4,5 & \\
\hline Brebis & 15000 litres & 2 & \\
\hline Vaches & 220000 litres & 5 & \\
\hline Vaches et brebis & 120000 litres & 3 & \\
\hline Bovins & $25-30$ têtes & 1,5 & Moins d' 1 an d'ancienneté \\
\hline Bovins & 50 têtes & 2 & \\
\hline Bovins & 170 têtes & 3 & \\
\hline Bovins et porcs & 80 têtes & 2 & \\
\hline Bovins & 120 têtes & 3 & \\
\hline Bovins & 100 têtes & 3 & \\
\hline Bovins & 40 têtes & 2 & Moins d' 1 an d'ancienneté \\
\hline Bovins & 20 têtes & 1 & Proche retraite \\
\hline Porcs & 1800 têtes & 16 & \\
\hline Bovins & 25 têtes & 1 & \\
\hline Bovins & 75 têtes & 2 & \\
\hline Bovins & 45 têtes & 1 & \\
\hline Bovins & 80 têtes & 1 & Moins de 1 an d'ancienneté \\
\hline Miel & 3 à 5 tonnes & 1 & En phase de transmission \\
\hline Miel & 8 tonnes environ & 2 & \\
\hline Maraichage & NC & 1 & 5 ans - Bio \\
\hline Maraichage & $7-10$ tonnes & 1 & Bio \\
\hline Maraichage & 20000 euros de CA & 1 & Bio et nature et Progrès \\
\hline Maraichage & 200 tonnes & 40 producteurs & Association \\
\hline Abattoir & 2300 tonnes & $\mathrm{NC}$ & \\
\hline $\begin{array}{l}\text { Atelier } \\
\text { transformation }\end{array}$ & $\begin{array}{l}200 \text { tonnes (ovins, } \\
\text { bovins, porcs, caprins) }\end{array}$ & 9 & $\begin{array}{c}\text { Développement récent } \\
\text { cosmétique, légumes en- } \\
\text { cours }\end{array}$ \\
\hline $\begin{array}{l}\text { Atelier } \\
\text { transformation }\end{array}$ & 190 tonnes & 8 & $\begin{array}{l}\text { Développement récent } \\
\text { charcuterie, en-cours : } \\
\text { plats cuisinés }\end{array}$ \\
\hline
\end{tabular}

\section{Elaboration personnelle}

\subsection{Caractéristiques du territoire et pratiques logistiques existantes}


Sur le plan géographique, la dynamique agricole de Saint-Flour Communauté se caractérise par une population de petits producteurs doublement isolés : (1) isolement géographique entre les zones de production, (2) éloignement des zones de forte demande. Le schéma 1 représente le plan du territoire, ces pôles urbains, et son principal réseau routier.

Figure 1 : Territoire de Saint-Flour Communauté, principaux pôles urbains et réseau routier principal

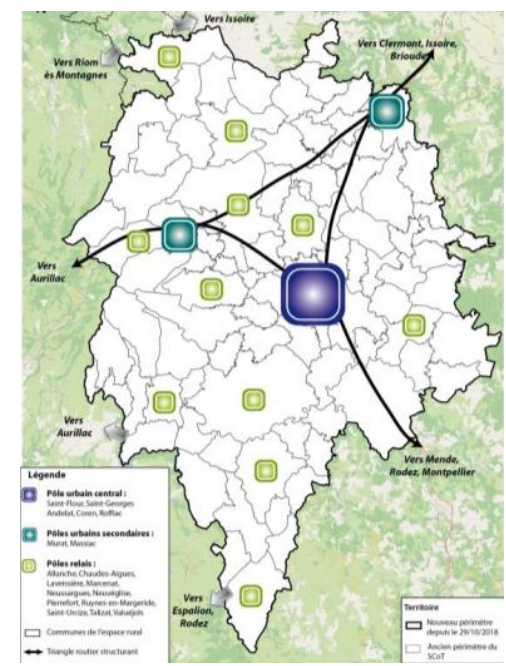

Source : Le Syndicat des Territoires de l'Est Cantal.

L'isolement et géographique des zones de production est dû au fait que le périmètre de ce territoire est au cœur d'un massif montagneux. Outre les massifs montagneux, ce sont des plateaux ou de profondes vallées qui complètent la diversité du paysage. Ce relief limite l'accessibilité de la zone à un axe Nord-Sud et fait obstacle au développement Est-Ouest du territoire, en rendant difficile toute traversée dans ce sens. Malgré un maillage fourni par l'existence de voies de communication, cette difficulté s'accentue lors des hivers enneigés.

La faible densité de la population, la ruralité sur les trois-quarts du territoire et l'altitude renforcent la complexité de la situation pour les producteurs. La conséquence de cette topographie de moyenne montagne est l'éloignement géographique dont souffrent les acteurs entre eux au sein du territoire et par rapport aux grands centres urbains à forte demande. Il faut, en effet, une heure pour rejoindre Clermont-Ferrand, métropole urbaine la plus accessible. Cependant l'ensemble du territoire bénéficie d'un bon réseau routier en étoile. 
Ainsi, hormis un secteur, la majorité des communes se situe à moins d'une heure de SaintFlour.

Les acteurs de Saint-Flour Communauté proposent une large diversité de produits que l'on peut regrouper en quatre sous-filières : viande, lait, miel, fruits et légumes. Cette diversité aux caractéristiques assez diverses (températures de conservation, taille des emballages, fragilité physique des produits...), a un impact direct sur la logistique (températures de stockage et de transport, risques de contamination en cas de mélange, délais d'approvisionnement divers...). Le tableau 3 suivant présente les principales données obtenues par filière.

Tableau 3 : Principales données obtenues par filière

\begin{tabular}{|c|c|c|}
\hline Filière & Contraintes/ freins circuits courts & $\begin{array}{l}\text { Organisation/ souhaits des acteurs } \\
\text { en circuits courts }\end{array}$ \\
\hline Viande & $\begin{array}{l}\text { Les ateliers de découpe = le goulot } \\
\text { d'étranglement de la filière }=>1 \mathrm{la} \text { « capacité } \\
\text { en volume traité par jour » la plus limitée } \\
\text { Dates de péremption = distribution rapide } \\
\text { après la découpe } \\
\text { Chaine du froid } \\
\text { Colisage ( } 5 \text { et } 10 \mathrm{kgs} \text { ) peu adapté à la } \\
\text { restauration, à la demande dans les grandes } \\
\text { villes (peu d'espace dans les frigo) } \\
\text { Une partie de la carcasse difficilement } \\
\text { valorisable en frais } \\
\text { Les transporteurs chers } \\
\text { Freins marketing (temps consacré à la } \\
\text { prospection commerciale, volumes } \\
\text { suffisants pour permettre l'abattage...) et } \\
\text { logistique (coûts de transport) }\end{array}$ & $\begin{array}{l}\text { Les pratiques d'élevage sont } \\
\text { hétérogènes. Les éleveurs tiennent à } \\
\text { garder cette autonomie } \\
\text { Les producteurs sont } \\
\text { particulièrement actifs sur le front de } \\
\text { la vente directe qu'ils souhaitent } \\
\text { majoritairement développer } \\
\text { Les abattoirs peuvent absorber } \\
\text { davantage de volumes } \\
\text { Les ateliers de découpe sont très } \\
\text { proches des éleveurs } \\
\text { Le véhicule frigorifique de la } \\
\text { collectivité joue un rôle déterminant } \\
\text { dans les livraisons sur le territoire }\end{array}$ \\
\hline $\begin{array}{l}\text { Lait/ } \\
\text { Fromage }\end{array}$ & $\begin{array}{l}\text { Concurrence forte (AOP/AOC) } \\
\text { Saisonnalité des ventes (Noël...) } \\
\text { Chaine du froid } \\
\text { Allotissement pour les circuits courts } \\
\text { (découpe et étiquetage) } \\
\text { Recours aux prestataires : coûteux ce qui } \\
\text { implique des minimas de commande }\end{array}$ & $\begin{array}{l}\text { Filière relativement structurée } \\
\text { autour des AOP/AOC } \\
\text { Tendance : autonomie de production } \\
\text { des fromages (ne plus passer par les } \\
\text { coopératives) et commercialisation } \\
\text { directe } \\
\text { Hétérogénéité dans le stockage et les } \\
\text { emballages } \\
\text { Transport en propre majoritaire, sauf } \\
\text { coopératives }\end{array}$ \\
\hline Miel & $\begin{array}{l}\text { Le coût du transport et, dans une moindre } \\
\text { mesure, le démarchage commercial = } \\
\text { principaux freins au développement des } \\
\text { acteurs hors territoire }\end{array}$ & $\begin{array}{l}\text { Ventes essentiellement sur le } \\
\text { territoire, mais perspectives au-delà du } \\
\text { territoire souhaitées et envisageables. } \\
\text { Activité saisonnière (Noël, période } \\
\text { touristique...) }\end{array}$ \\
\hline Maraîchage & $\begin{array}{l}\text { L'hétérogénéité des normes } \\
\text { (conventionnel, Bio...) => pas de mélanges } \\
\text { possibles }\end{array}$ & $\begin{array}{l}\text { La lentille blonde de Saint-Flour: } \\
\text { très structuré (association de } \\
\text { producteurs) : volumes importants, } \\
\text { ventes essentiellement hors du } \\
\text { territoire, contrat de transport } \\
\text { Reste de la filière concentrée sur les } \\
\text { circuits courts dans le territoire, forte } \\
\text { demande => pas de volonté d'aller } \\
\text { hors territoire } \\
\text { Transport en propre, peu optimisé }\end{array}$ \\
\hline
\end{tabular}

Source : Elaboration personnelle 
Les producteurs se heurtent à une vive concurrence intra territoriale due à un faible volume de demande du fait de la ruralité et de la faible densité de population. Au-delà du territoire, la demande est plus forte, mais les acteurs se heurtent alors à des difficultés quant l'organisation de la logistique et aux coûts de transport. Sur le territoire, il existe plusieurs solutions de transport : le transport en propre (avec le véhicule du producteur), la location d'un véhicule réfrigéré à la Communauté de communes, ou le recours à des prestataires. Avec ces derniers, les producteurs individuels ont souvent du mal à fournir un colis qui réponde à la condition minimale de poids imposée. De même en ce qui concerne les volumes à regrouper pour rentabiliser la location d'un véhicule.

L'attraction de la vente directe est certaine pour les acteurs du territoire mais sa mise en œuvre est complexe et exige une organisation spécifique. De manière générale, les producteurs fournissent, en majorité, des produits dans un rayon de $100 \mathrm{kms}$ autour de SaintFlour pour des raisons de temps et d'argent. Les acteurs se heurtent à de nombreuses limites dans les circuits courts : (1) les flux individuels sont généralement trop faibles pour absorber les frais de transport hors territoire ; (2) à l'intérieur du territoire, une meilleure organisation logistique permettrait de diminuer les coûts de transport et de gagner du temps dans les exploitations.

\section{Solution organisationnelle et technique et discussion des résultats}

\subsection{La frontière de l'action du collectif et son organisation}

Les producteurs sont favorables à la mise en place d'une démarche collective de mutualisation des transports à condition que la mise en place de cette solution n'est pas d'effets marketing et commercial sur la clientèle déjà acquise par chacun des producteurs. Les craintes se situent à deux niveaux : la préservation de leur portefeuille de clientèle actuel (les producteurs rejettent toute idée de mutualisation des clients); la relation directe avec les clients (l'outil collectif ne doit pas faire écran entre les producteurs et le marché). Il semble donc que la frontière du domaine d'action du collectif soit d'ordre logistique mais que les acteurs rejettent tout rapprochements commerciaux (Cassière et Noireaux, 2020). Paradoxalement, pour une grande majorité d'acteurs, la problématique principale est la survie ou le développement commercial. Mais le risque d'opportunisme perçu dans la relation coopétitive est trop important pour permettre une stratégie commerciale commune, surtout 
chez les plus petits d'entre eux. En effet, si les acteurs veulent bien collaborer sur la dimension logistique, certains sont également concurrents. La peur d'un détournement commercial par le partenaire est très présente, et ce, entre concurrents directs (producteurs de viande bovine bio par exemple), indirects (producteurs bio versus conventionnels) et intrafilières (producteurs ovins et bovins).

Au-delà de la frontière du collectif, se pose la question de la viabilité du projet. Ce dernier ne peut se faire sans un pilotage logistique inhérent à l'organisation mise en place (Rouquet et Vauché, 2015). Ce pilotage, incarné par l'acteur pivot devra assurer la coordination des opérations sur le long terme. En logistique, le pilotage de l'organisation peut être incarné un acteur individuel de la chaine logistique (producteur, PSL...) ou par un collectif de parties prenantes. Le pilote doit posséder certains attributs reconnus par les autres membres, tels que les compétences nécessaires, la légitimité d'organiser les flux, et il doit incarner des objectifs communs (Noireaux et Ralet, 2019). Du fait des attributs attendus, les consommateurs sont généralement écartés d'emblés d'un éventuel pilotage logistique, pour des raisons de compétences et parfois de légitimité.

En logistique, le rôle joué par le PSL est un facteur clé de succès des démarches de mutualisation (Rouquet et Vauché 2015). Dans nos résultats, le PSL ne représente pas un acteur pivot potentiel, car leur position concurrentielle, quasi-monopolistique sur le territoire, leur confère un important pouvoir face aux agriculteurs. Cette situation a déjà été identifié dans la littérature, où la trop grande dysmétrie de pouvoir écarte le PSL du pilotage (Noireaux et Poirel, 2014).

Dans notre étude, aucun acteur individuel n'a souhaité endosser ce rôle pour des raisons de compétences et de temps, préférant se concentrer sur leur cœur de métier.

En matière de stratégies collectives (Noireaux et Poirel, 2014), l'acteur pivot peut être représenté par une structure (souvent associative). Dans nos résultats, cette solution est peu envisagée par les acteurs. Les problèmes liés à la gestion du temps et à l'inégalité perçue dans l'implication du collectif sont les principaux arguments mis en avant.

Le collectif souhaite que l'acteur pivot soit la collectivité territoriale. En effet, cette dernière parait légitime du fait de son implication forte dès le début du projet. Par ailleurs, elle a la volonté de soutenir et d'accompagner les acteurs dans la mise en place de solutions. Les acteurs publics, et en particulier les collectivités sur certains territoires fragiles, constituent un levier déterminant de l'émergence de stratégies collectives (Audretsch et al., 2019). 
Cependant, dans notre cas, si la collectivité a pour vocation de participer au développement de solutions (mise à disposition d'un local, aide financière pour les investissements...), il n'est pas envisageable qu'elle engage à long terme de la masse salariale ou des frais fixes, pour des raisons politiques, car les choix de fléchage des budgets peuvent changer en fonction des programmes des élus.

\subsection{La solution technique retenue}

Techniquement, deux solutions de transport complémentaires doivent être développées. Les deux solutions s'appliquent différemment, selon qu'on commercialise proche du territoire de Saint-Flour communauté (moins de $100 \mathrm{kms}$ ) ou qu'on expédie sa marchandise hors du Cantal (Montpellier, Paris Clermont-Ferrand, Béziers...). Nos résultats sont donc concordant avec les travaux du PIPAME (Pôle interministériel de prospective et d'anticipation des mutations économiques) où il existe deux types d'organisation logistique territoriale (Blanquart et al., 2009). La logistique exogène et la logistique endogène. La logistique endogène est fondée sur l'activité de production réalisée dans le territoire et couvre l'activité de consommation au sein de ce territoire. La logistique exogène repose sur des besoins d'organisations logistiques extérieures au territoire local et correspond à une logistique géographiquement étendue. Ces solutions apparaissent parce que nous n'avons pas pris en compte la proximité géographique dans notre définition des circuits courts, ce qui complexifie le design de l'organisation logistique.

Figure 2 solutions logistiques possibles 


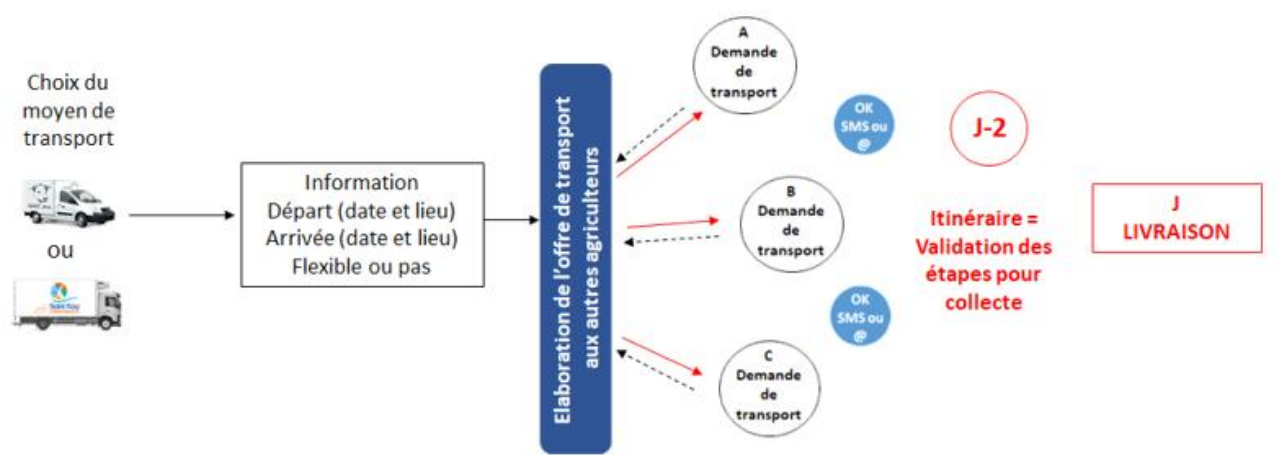

Scenario 2 Trajet supérieur à 100 kms - Généalogie des demandes de mutualisation et colisage.

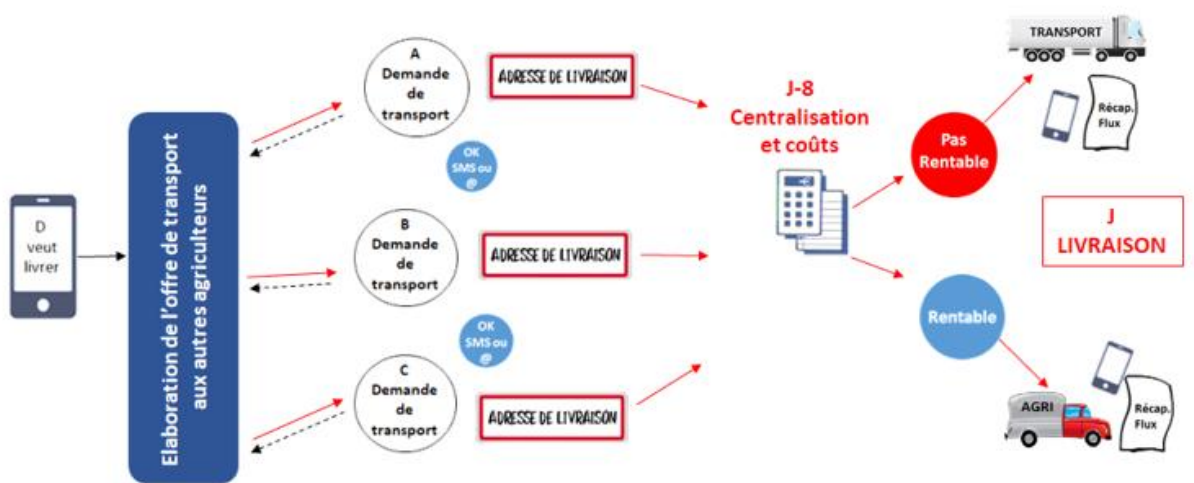

\section{Solution de transport proche (moins de $100 \mathrm{kms}$ )}

La solution retenue est basée sur le principe du covoiturage. La solution consiste à compléter son chargement avec les marchandises d'autres producteurs ou personnes situés sur son itinéraire et desservant le même point final ou un point proche de celui-ci. Leurs succès sont assurés grâce à la démocratisation des outils des “Technologies de l'Information et de la Communication" (TIC), notamment à travers les smartphones et les fonctionnalités de géolocalisation (Evans et Schmalensee, 2017). Ces initiatives incitent à une meilleure occupation des véhicules. Elles sont largement diffusées dans les systèmes de mobilité humaine. Le nouveau défi serait d'introduire une telle architecture dans le transport des marchandises.

L'objectif de cette solution est de donner de la visibilité à chacun sur les tournées réalisées. Deux types de tournées peuvent-être proposés : les tournées fixes et les tournées ponctuelles. Chaque producteur est alors libre de s'agréger à l'offre de transport proposée. La coordination doit se faire intuitu personae par appel direct... Le frein que peut représenter l'acteur pivot est 
résolu par l'utilisation de la plateforme numérique (Evans et Schmalensee, 2017). Ce projet s'est inscrit dans un projet plus large de plate-forme de mobilité sur le territoire. La Région et Saint-Flour Communauté développent en effet un projet de mobilité des personnes en territoire rural. Le projet logistique a été intégré à ce projet plus global en février 2020. Cette plateforme doit être livrée par le prestataire en septembre prochain.

Pour répondre à la contrainte de diversité des flux, une solution de bacs individuels fermés de taille standard pour chaque producteur a été retenue. Avec cette solution, les producteurs peuvent transporter différents types de marchandises dans un même véhicule, sans risque de contamination tout en préservant les impératifs de diversité des températures de transport exigées. La solution n'exige pas que les producteurs distribuent aux mêmes clients, il faut juste qu'ils aillent vers les mêmes destinations. On transporte pour le compte du voisin, et en retour les frais de transport sont partagés, on économise les charges de carburant et l'on gagne en temps. Il existe un ensemble de calculs simples qui conduisent à la fixation du coût par caisse et par km effectué. L'identification des points de regroupement ou de collecte se traite par téléphone et de gré à gré.

Au-delà de l'aspect technique et financier, cette solution permet aux acteurs de se projeter dans davantage de coopérations et d'entre-aides sur le territoire. Ici, les outils représentent le socle d'une collaboration souhaitée et naissante, mais où la méfiance reste encore présente. Cette dernière est traduite dans les outils. La rétribution du transport est vue par les acteurs comme un bouclier à certains comportements opportunistes potentiels qui chercheraient uniquement à profiter des transports d'autrui sans jamais apporter de retour.

\section{$\underline{\text { Solution de transport lointain (plus de } 100 \mathrm{kms} \text { ) }}$}

Pour la commercialisation hors Cantal, il faut rentabiliser les transports en ciblant des zones à forte demande (Montpellier, Paris, Béziers et Clermont-Ferrand). Le problème actuel est lié à de faibles volumes transportés qui engendrent des coûts de transport importants. L'absence d'une prospection commerciale ciblée et articulée a pour conséquence l'éparpillement du flux. Cette situation est vécue comme une véritable problématique par les agriculteurs et limite les ventes hors territoire.

Une solution de négociation collective de contrat de prestation a été retenue. Ce dispositif de mutualisation consiste à négocier un contrat collectif avec un prestataire privé pour une période donnée (généralement un ou deux ans). Cette solution se fonde sur le pouvoir de 
négociation des acteurs et ne peut être envisageable en dehors d'une démarche collective concertée (Noireaux, 2006). La structure commune négocie les prix avec un prestataire. Chaque acteur contractualise ensuite directement sur la base négociée.

Cette solution est envisageable sur le territoire à partir du moment où les prérequis suivants sont remplis :

1/ L'adhésion des acteurs les plus structurés : les quelques agriculteurs du territoire qui utilisent déjà des prestataires doivent être parties prenantes de la démarche. En effet, le regroupement d'acteurs ayant déjà des volumes relativement importants permet de mieux négocier au départ en s'engageant sur un historique de livraison. Ce critère a été accepté par les quatre principaux producteurs du territoire. Ils ont également accepté de participer au comité de pilotage de la structure commune, et la Communauté de commune s'est engagée à baliser $10 \%$ du temps de travail du chargé de mission agricole pour traiter de cette problématique (mention voté en 2020 pour 3 ans).

2/ La prospection commune sur des villes cibles: Il existe une réelle volonté de se développer hors du territoire cantalien. Cette volonté doit être traduite par la mise en place d'une démarche commerciale via une personne dédiée (de type agent commercial) qui présenterait, dans les villes visées (Paris, Lyon et Montpellier), l'ensemble de la production du territoire. Ce premier contact se traduit par la mise en place d'un fichier commun permettant à chacun des agriculteurs de négocier et de vendre ses produits. Néanmoins, la mise en place d'un commercial pour toute la zone doit veiller à respecter un principe de neutralité et de transparence maximales fin d'éviter tout amalgame ou opportunisme au profit d'acteurs particuliers et au détriment de l'unité collective. La Chambre d'Agriculture a proposé d'embaucher un chargé de mission afin de réaliser cette tâche. Cette proposition est en attente de validation.

Pour être efficace et rentable, la solution doit grouper le nombre de points de collecte et les tournées de livraison. Autrement dit, il faut une concentration des flux par destination et un calendrier des tournées à respecter. La marchandise doit être centralisée chez un prestataire de la région ou sur un point central proche de Saint-Flour.

Il faut souligner que cette démarche est réalisable dans une optique de long terme. En effet, si les contrats mutualisés sont présents dans d'autres secteurs, il faut en moyenne entre 5 et 7 ans pour réaliser et consolider totalement la démarche (Noireaux, 2006). 


\section{Conclusion}

Cette recherche a permis de valider à l'échelle d'un territoire rural la difficile construction d'une démarche collective tant les attendus et les spécificités des acteurs sont particuliers. Si les acteurs s'accordent à reconnaître une nécessaire harmonisation des pratiques pour atteindre la performance logistique, nombre d'entre eux ne sont pas forcément prêts à s'investir, à partager pour une œuvre collective. La coopération est un état souhaité, mais les acteurs restent méfiants quant à son équilibre : équilibre dans les efforts pour collaborer, et équilibre dans les bénéfices de la collaboration.

Les deux solutions logistiques présentées permettent de mettre l'accent sur quelques facteurs clé de succès à la réussite de ces designs logistiques. Pour le modèle de proximité (moins de $100 \mathrm{kms}$ ) l'implication individuelle au service des parties prenantes montre qu'il repose sur un fort intuitu personae entre acteurs. Il peut être d'une extrême souplesse tant que les acteurs s'accordent pour assurer la fluidité des livraisons. Le modèle à plus de $100 \mathrm{kms}$ est plus contraignant en termes de structure et d'architecture mais permet en contrepartie de générer des volumes d'affaires plus conséquents en regroupant l'amont. Il repose aussi sur une forte confiance mutuelle car la remise dans les mains d'un tiers mandaté de la prospection commerciale sur une zone définie fait planer une incertitude chez les producteurs quant à ce que qu'il peut être fait des informations qu'ils fournissent. Le spectre de l'opportunisme est fortement présent dans cette architecture. Tout au long de l'élaboration du modèle, la méfiance les a amenés à mettre en place des outils de contrôle de la collaboration (rétribution financière, visibilité de tous sur le futur prospect, refus d'une pilotage privé...). Cette recherche action nous a permis de définir et de préciser le design logistique des circuits courts dans un territoire rural. Dans le futur, nous allons suivre la mise en place des deux modèles identifiés (proximité et plus lointain). Nous allons également confronter notre design logistique avec d'autres territoires de caractéristiques similaires ou proches.

\section{Bibliographie}

Agreste Enquêtes structures ESEA (2016), https://agreste.agriculture.gouv.fr/agrestesaiku/?plugin=true \&query=query/open/ESEA16_4

Aoki, M. (1990). Toward an economic model of the Japanese firm. Journal of economic literature, 28(1), 1-27. 
Audretsch D.B., Link A.N., \& Scott J.T. (2019), Public/private technology partnerships: evaluating SBIR-supported research, in The Social Value of New Technology. Edward Elgar Publishing.

Blanquart C., Kebir L., Petit C. \& Traversac J.B. (2009), Les enjeux logistiques des circuits courts, rapport pour le PIPAME.

Bonomelli V. \& Roudart L. (2019), Quels effets des circuits courts de commercialisation sur les moyens d'existence des agriculteurs familiaux ? Le cas d'une foire paysanne à Quito (Équateur). Économie rurale 367 (1), p. 95-111.

Cassière F., Noireaux V. \& Edzengde Edzengte J. (2020), Stratégie collective logistique des agriculteurs : une difficile équation, $13^{\text {èmes }}$ Rencontres Internationales en Logistique et Supply Chain Management, 7-9 octobre, Le Havre.

Chiffoleau Y. (2019), Les circuits courts alimentaires Entre marché et innovation sociale, ERES «Sociologie économique » [en ligne] https://www-cairn-infoezproxyucafr/lescircuits-courts-alimentaires--9782749262345htm.

Chiffoleau, Y., \& Paturel, D. (2016). Les circuits courts alimentaires «pour tous», outils d'analyse de l'innovation sociale. Innovations, (2), 191-210.

Dari, L. \& Paché G. (2015), Acteurs tiers et stratégies collectives au sein des filières. Le cas du liège en Corse, Économie rurale. Agricultures, alimentations, territoires 349-350, p. 101-123.

Evans D. \& Schmalensee R. (2017), De précieux intermédiaires : comment BlaBlaCar, Facebook, PayPal ou Uber créent de la valeur. Odile Jacob.

Krief, N., \& Zardet, V. (2013). Analyse de données qualitatives et recherche-intervention. Recherches en sciences de gestion, (2), 211-237.

Maréchal, G. (2008). Les circuits courts alimentaires: bien manger dans les territoires. Educagri éditions.

Masson S. \& Petiot R. (2013), Logistique et territoire: multiplicité des interactions et forces de régulation, Géographie, économie, société, (15) 4, p. 385-412.

Moati P. (2018), (Dé) penser la consommation : peut-il y avoir une «bonne consommation ? Éditions EMS.

Moisdon, J. C. (2015). Recherche en gestion et intervention. Revue française de gestion, 41(253), 21-39.

Mundler, P., \& Laughrea, S. (2015). Circuits alimentaires de proximité-Quels bénéfices pour le développement des territoires? Étude de cas dans trois territoires québécois (No. 2015rp-21). CIRANO. 
Munzele Munzimi J-M. (2013), “L'alimentation comme fondement de l'identité culturelle, Mouvements et Enjeux sociaux, no 76, janvier-février, pp. 15-30.

Noireaux V. \& Poirel C. (2014), Contrepouvoir et stratégies collectives : comment contrer le pouvoir d'un puissant partenaire dans le canal de distribution ? Revue Française de Gestion, 243(40), p. 81-95.

Noireaux V. \& Ralet P. (2019), Difficultés des canaux de distribution alimentaire en territoire rural : l'importance du leader, Économie rurale 369 (3), p. 21-39.

Noireaux V. (2006), L'émergence du leadership reconnu dans le canal de distribution inversée: le cas des déchets industriels (Doctoral dissertation, Aix-Marseille 2).

Paciarotti C. \& Torregiani F. (2021), The logistics of the short food supply chain: a literature review, Sustainable Production and Consumption, 26, p. 428-442.

Pouzenc M. (2012), Les grandes surfaces alimentaires contre le territoire... tout contre. Pour, 3 , p. 255-261.

Raulet-Croset, N. (2014). Le territoire comme objet de gestion. In Annales des MinesResponsabilité et environnement (No. 2, pp. 48-52). ESKA.

Requier-Desjardins D, Roudart L (2019) Introduction Les circuits courts alimentaires : regards croisés entre l'Amérique latine et l'Europe occidentale. Économie rurale 367 (1) : 7-11 [en ligne] https://www-cairn-infoezproxyucafr/revue-economie-rurale-2019-1-page$7 \mathrm{htm}$

Rouquet A., \& Vauché L. (2015), A typology of logistics pooling in supply chains, Supply Chain Forum: An International Journal, 16 (2), p. 2-12.

Vaillant L., Gonçalves A., Raton G. \& Blanquart C. (2017), Transport et logistique des circuits courts alimentaires de proximité: la diversité des trajectoires d'innovation, Innovations, 3, p. 123-147. 